\title{
Intraoperative ultrasonographic localization of pulmonary ground-glass opacities
}

\author{
Ryoichi Kondo, MD, PhD, ${ }^{\mathrm{a}}$ Kazuo Yoshida, $\mathrm{MD}, \mathrm{PhD},{ }^{\mathrm{a}}$ Kazutoshi Hamanaka, MD, ${ }^{\mathrm{a}}$ \\ Masahiro Hashizume, MD, ${ }^{\text {a }}$ Toshiki Ushiyama, MD, ${ }^{\mathrm{a}}$ Akira Hyogotani, MD, ${ }^{\mathrm{a}}$ Makoto Kurai, MD, ${ }^{\mathrm{a}}$ \\ Satoshi Kawakami, MD, ${ }^{\mathrm{b}}$ Mana Fukushima, $\mathrm{MD},{ }^{\mathrm{c}}$ and Jun Amano, MD, PhD ${ }^{\mathrm{a}}$
}

\begin{abstract}
Objectives: Ground-glass opacities are typically difficult to inspect and to palpate during video-assisted thoracic surgery. We therefore examined whether ultrasonographic assessments could localize ground-glass opacities and help to achieve adequate resection margins.
\end{abstract}

\begin{abstract}
Methods: An intraoperative ultrasonographic procedure was prospectively performed on 44 patients harboring ground-glass opacities of less than $20 \mathrm{~mm}$ in diameter to localize these lesions and to achieve adequate margins. We also examined whether there were any complications resulting from the intraoperative ultrasonogram, such as lung injury, heart injury, or arrhythmia. We excluded patients with both asthma and chronic obstructive pulmonary disease from this study inasmuch as the intraoperative ultrasonographic procedure is more difficult to interpret when residual air is present in the lung.
\end{abstract}

Results: A total of 53 ground-glass opacities were successfully identified by intraoperative ultrasonography without any complications. Of the 20 mixed ground-glass opacities that we examined, 15 were found on palpation. However, only 4 (12.1\%) of the 33 pure ground-glass opacities could be palpated. In all instances in which complete collapse of the lung was achieved (30/53 of these cases), high-quality echo images were obtained. Additionally, a strong correlation was found between the resection margins measured by ultrasonogram and the margins determined by histologic examination in the resected lung specimens $\left(r^{2}=0.954, P<.001\right)$.

Conclusions: Intraoperative ultrasonography can both safely and effectively localize pulmonary ground-glass opacities in a completely deflated lung. This procedure is also useful for the evaluation of surgical margins in a resected lung. Hence, ultrasonography may assist surgeons to perform minimally invasive lung resections with clear surgical margins during the treatment of solitary lung ground-glass opacity.

Over the past 10 years, solitary pulmonary nodules have been detected more frequently owing to the development of computed tomography (CT) scanners and the more widespread use of a chest CT in health screens. Most solid lung nodules can be identified easily by palpation, but many ground-glass opacities (GGOs) cannot be so readily detected during surgical procedures such as video-assisted thoracic surgery (VATS). ${ }^{1}$ Hence, the establishment of techniques that can confirm the location of these tumors in collapsed lungs during surgery and assist clinicians in determining the margin of the resected lung from the GGO is of some importance.

The use of intraoperative ultrasonography (IU) during lung surgery has stemmed from the experiences of surgeons who perform abdominal procedures. ${ }^{2-5}$ Importantly, this tech-

\footnotetext{
From the Departments of Surgery, ${ }^{\mathrm{a}}$ Radiology, ${ }^{\mathrm{b}}$ and Pathology, ${ }^{\mathrm{c}}$ Shinshu University School of Medicine, Matsumoto, Japan.

Received for publication May 28, 2008; revisions received Dec 20, 2008; accepted for publication Feb 2, 2009; available ahead of print March 27, 2009.

Address for reprints: Ryoichi Kondo, MD, PhD, Department of Surgery, Shinshu University School of Medicine, 3-1-1 Asahi, Matsumoto 390-8621, Japan (E-mail: kryoichi@shinshu-u.ac.jp).

J Thorac Cardiovasc Surg 2009;138:837-42

$0022-5223 / \$ 36.00$

Copyright (c) 2009 by The American Association for Thoracic Surgery

doi:10.1016/j.jtcvs.2009.02.002
}

nique has now made it possible both to detect small nodules in the lung and to evaluate whether the lines of resection will achieve the required margins for solid lung nodules. ${ }^{3,4,6-15}$ Although the utility of IU for the management of GGO has remained unclear, we hypothesized that this procedure would enable the identification of small GGO lesions deep in the parenchyma and could serve as a guide in finding the appropriate margin in a deflated lung.

\section{PATIENTS AND METHODS}

In our hospital, we performed IU on patients for whom this procedure was deemed appropriate and evaluated whether we could localize GGOs and achieve an adequate resection margin using this method. We previously conducted a power analysis using a literature search for IU treatment of solid lung nodules in 2001..$^{6-9,11,12}$ The results of this analysis suggested that we would need 35 cases to evaluate the usefulness of IU for treating GGO, and our current clinical trial was designed accordingly.

\section{Subjects \\ Between January 2002 and December 2007, 153 patients in whom a sol- itary GGO of within $20 \mathrm{~mm}$ in diameter was detected by chest CT were re- ferred to Shinshu University Hospital. A VATS resection was carried out in 96 of these patients because of a strong suspicion of lung cancer. We re- cruited 44 of these 96 patients who had no history of asthma, chronic ob- structive pulmonary disease, pulmonary fibrosis, or severe heart disease for our present study (Figure 1). The IU procedure requires no residual air, and patients who have chronic obstructive pulmonary disease or asthma}




\section{Abbreviations and Acronyms \\ $\mathrm{CT}=$ computed tomography \\ $\mathrm{GGO}=$ ground-glass opacity \\ $\mathrm{IU}=$ intraoperative ultrasonography \\ VATS $=$ video-assisted thoracic surgery}

are more likely to have trapped air in their lungs. In addition, in patients with pulmonary fibrosis or severe heart disease, it is often difficult to maintain single-lung ventilation during VATS. Informed consent was obtained from each patient and both the experimental protocols and patient consent forms were approved by the University of Shinshu School of Medicine Committee on the Protection of the Rights of Human Subjects.

This subject group comprised 19 men and 25 women with an age range of 31 to 80 years (mean $62.8 \pm$ SD 11.2). A chest CT scan showed no evidence of mediastinal disease or pleural thickness that would have indicated severe adhesions in any of the patients. The respiratory functions of these subjects were also found to be within the normal range (forced expiratory volume in 1 second, $2147 \pm 668 \mathrm{~mL}$; forced vital capacity, $2806 \pm 906$ $\mathrm{mL}$; percent forced expiratory volume in 1 second, $78.9 \% \pm 7.1 \%$; percent vital capacity, $103.2 \% \pm 16.9 \%$ ). The sizes of the GGOs were all less than or equal to $20 \mathrm{~mm}$ in diameter, as determined by preoperative high-resolution CT. Among the cohort we tested, 38 patients showed a solitary GGO and the remaining 6 had multiple GGOs: 4 patients had 2 GGOs, 1 patient had 3 GGOs, and 1 individual had 4 GGOs. A total of 53 GGOs were thus detected among these 44 individuals who subsequently underwent VATS. These lesions comprised 33 pure and 20 mixed GGOs that showed a small solid component in the central region. A preoperative hook-wire technique was used to localize pure GGOs that were less than $10 \mathrm{~mm}$ in diameter and did not involve the visceral pleura. This additional measure was taken because these lesions are nearly impossible to identify in the event that the lung does not deflate completely.

\section{Ultrasonogram}

We used the Diagnostic Ultrasound System SSD-2000 (Aloka Instruments, Tokyo, Japan) in this study, which consists of a probe with a flexible tip (UST-5536-7.5) and has a linear array transducer with a frequency of 7.5 MHz. The dimensions of the probe are $10 \mathrm{~mm}$ in diameter and $38 \mathrm{~cm}$ in length.

\section{IU Technique}

All of our subject patients received general anesthesia using a double-lumen endotracheal tube. Single-lung ventilation was initiated as soon as the patient had been rotated into the lateral decubitus position so that the lung would have collapsed by the time the thoracoscope was inserted into the pleural space. After ensuring that there were no pleural adhesions by thoracoscopic examination, we set up the probe used to generate the ultrasonogram. The first palpation of the lung using the instrumental probe was carried out in the region where the lesion was thought to reside on the basis of CT scanning. When the lung had been collapsed completely, the probe was placed firmly in the area of the target lesion. The pleural space was filled with $500 \mathrm{~mL}$ of warm saline to assist in obtaining a better definition IU view. If the complete collapse of the lung could not be achieved, careful suction of the airways or application of gentle pressure to the lung surface with the IU probe was added to reduce residual air. The IU images were then video recorded at a frequency of either 7.5 or $18 \mathrm{MHz}$. We judged that the lung was completely deflated when its structure could be confirmed by a depth of 3 $\mathrm{cm}$ or more (the length from the visceral pleura). In completely deflated lungs, the structures of the pulmonary arterioles and venules are revealed as homogeneous hypoechoic areas, whereas the bronchioles appear as hyperechoic spots, in the vicinity of the pulmonary arterioles (Figure 2).

On achieving a completely deflated lung, we evaluated whether the target GGO could be identified by IU. If the target lesions were positively identified, their sizes, echo levels, and posterior echo were recorded. The echogenicities of internal GGOs were compared with those of the surrounding normal lung parenchyma. Posterior echo criteria were defined by comparisons with the echo levels of the surrounding lung parenchyma.

To ensure a sufficient surgical margin during the IU view of the GGO, we used electrocautery to mark the visceral pleura and to define the margins of intended resection (Figure 3). In cases involving a deeply localized GGO, an evaluation of the relationship between the GGO and nearby pulmonary artery, vein, and bronchus was included using the aforementioned procedure (Figure 4). When a wedge resection or segmentectomy was carried out, we determined whether the distances of the GGO from the resected margins

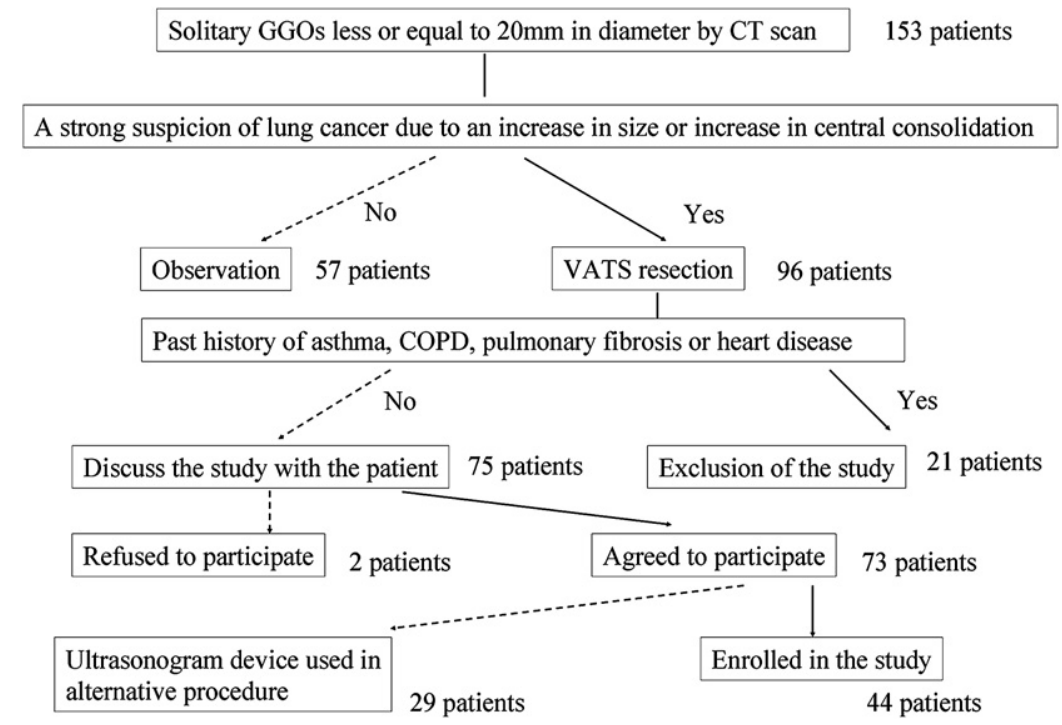

FIGURE 1. A CONSORT flow diagram for the criteria used to enroll patients in this study. Twenty-nine of the patients who agreed to participate could not subsequently be examined by intraoperative ultrasonography because the ultrasonogram device was unavailable. Forty-four patients were eventually enrolled. $G G O s$, Ground-glass opacities; $C T$, computed tomography; VATS, video-assisted thoracic surgery; $C O P D$, chronic obstructive pulmonary disease. 


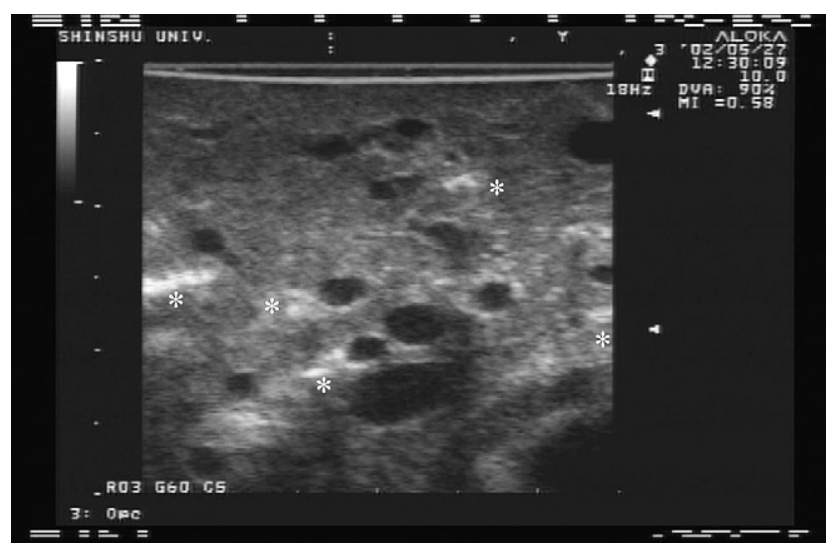

FIGURE 2. Intraoperative ultrasonographic image of an entirely deflated lung in which the structures of the lung parenchyma could be visualized. The pulmonary arterioles and venules revealed a homogeneous hypoechoic area. Asterisks indicate bronchioles that appear as hyperechoic spots in the vicinity of the pulmonary arterioles.

using the resected lung specimens could be measured by IU. If we succeeded in measuring the resection margins by IU, these distances were compared with those obtained for the same lesion via histologic examination. We considered the resection margin as "adequate" when the distance from the lesion to the resection margin was 1.5 times the maximum diameter of the GGO or $2 \mathrm{~cm}$.

In each case we assessed whether complications such as lung injury, heart injury, or arrhythmia arose from the IU procedure.

\section{Statistical Analysis}

The measured GGO sizes were compared by either an analysis of variance with corrections for multiple comparisons (Scheffé test) or by $t$ tests as appropriate. Categorical data were analyzed by $\chi^{2}$ tests. Relationships among variables were determined with Pearson correlation coefficients. The data are expressed as the mean value \pm the standard deviation.

\section{RESULTS}

No complications were found from the use of IU in any of our 44 patients. Although 15 (76.2\%) of the 20 mixed GGOs could be palpated, this was the case for only $4(12.1 \%)$ of the 33 pure GGOs (Table 1). Surgical resection of the GGO was subsequently performed by wedge resection in 23 cases, segmentectomy in 20 cases, and lobectomy in 10 cases. From the resulting diagnoses, we found that these cancers comprised 45 adenocarcinomas, 6 examples of atypical adenomatous hyperplasia, and 2 fibrotic benign nodules.

All 53 GGOs among our patient cohort were successfully identified by IU. A completely collapsed lung could be achieved in 30 of these 53 cases, and this enabled a high quality ultrasonogram image to be obtained. Although a preoperative hook-wire technique was used for 13 of these lesions, the lesions could all be identified by ultrasonogram. Nevertheless, even when the hook-wire marker was placed near the GGO, its presence did not prevent a discriminatory examination of an intrapulmonary GGO.

The internal echo images of the GGOs were revealed by high echo in 46 cases and low echo in the remaining 7 cases. The GGO types determined by echo imagery did not correlate with their size, histologic type, or presence of solid components. However, the echo types did strongly correlate with a complete deflation of the lung (Table 2). In all patients in whom the complete collapse of the lung was achieved, the GGO produced clear high echo images. On the other hand, in patients in whom a complete deflation of the lung could not be obtained, 9 GGOs showed high echo images and another 7 generated low echo images. In 8 cases, the target GGO appeared as a low echo image during the initial incomplete deflation period. At full collapse in these cases, the same GGO could be detected as a clear high echo image. The size range of the GGOs in our patient cohort, determined by IU, was $9.9 \pm 3.5 \times 5.4 \pm 2.1 \mathrm{~mm}$. These sizes were significantly smaller than those measured by CT $(12.1 \pm 3.8 \times$ $10.4 \pm 3.3 \mathrm{~mm}$ ).

The posterior echoes of the target lesions were found to be attenuated in 36 cases, accentuated in a further 7 lesions, but

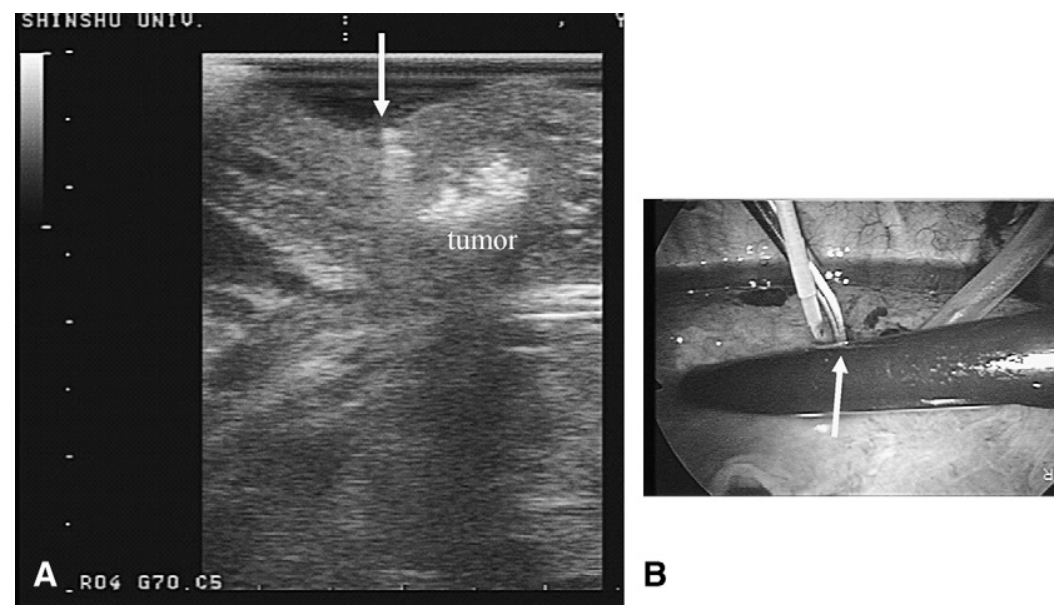

FIGURE 3. A, Intraoperative ultrasonographic image of a ground-glass opacity. The white arrow indicates forceps placed near to the edge of the tumor. B, Electrocautery marking on the visceral pleura was performed around the target lesions using ultrasonographic views of the ground-glass opacity. 

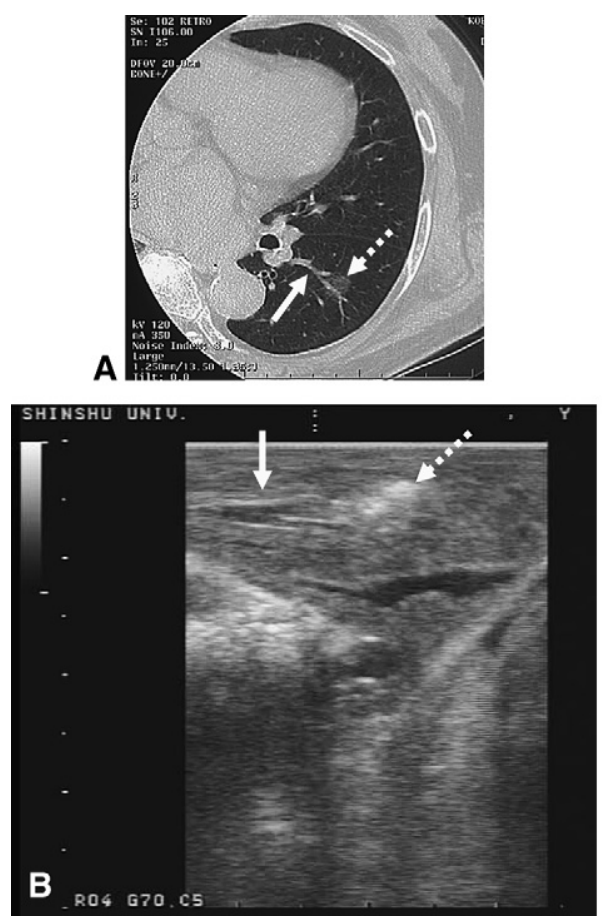

FIGURE 4. The relationship between a ground-glass opacity (dashed arrow) and nearby pulmonary artery (A and B, white arrow) or bronchus (C and D, white arrow).

showed no change in 10 cases. Moreover, all instances of acoustic shadow were attributed to high internal echo images, whereas all of the acoustic enhancements that we detected were ascribed to the presence of low internal echo images. The average maximum diameter of the GGO that had posterior echoes was significantly larger than the diameters of those that had no posterior echoes (Table 3).

The resected margins were evaluated in 23 GGOs by wedge resection and in 20 GGOs by segmentectomy. In all cases in which a wedge resection was performed, the distance of the tumor resected margin could be successfully measured by ultrasonogram. On the other hand, the distances from the resected edges to the GGO could be evaluated in only 11 cases in which a segmentectomy was performed. In the resected lung specimens, there was a strong correlation between the resected margins measured by ultrasonogram and the margins determined by histologic examination $\left(r^{2}=0.954, P<.001\right)$.

\section{DISCUSSION}

In our present study, the location of 53 GGOs could be successfully determined by ultrasonography. Previous studies have reported no complications arising from the use of $\mathrm{IU},{ }^{1,7,14,15}$ and we found this to be the case also in our present study. In addition, the successful location of pulmonary solid lung nodules by IU is $94.0 \% \pm 7.0 \%$ on the basis of current reports. ${ }^{3,4,6-15}$
Many solid lung nodules appear in a hypoechoic pattern, with an associated acoustic enhancement of the posterior echo. These solid tumors are thus easily visualized, even in lungs that have been incompletely deflated. ${ }^{7}$ In our current study, all of the GGOs analyzed displayed a hyperechoic pattern in a completely deflated lung. The ultrasonographic GGO images that could be obtained under incomplete deflation were lower in clarity than those generated for a completely deflated lung. If the lung had not been sufficiently deflated, however, the structures of interior lungs were likely to display a "spotted hyperechoic pattern" owing to residual air echo artifacts, and this can mask the presence of a GGO. The complete collapse of the lung is therefore necessary for the reliability of this procedure.

TABLE 1. Relationship between GGO type and the procedures used to treat the corresponding patients

\begin{tabular}{|c|c|c|c|c|}
\hline & Total no. & Pure GGO & Mixed GGO & $P$ value \\
\hline \multicolumn{5}{|c|}{$\begin{array}{l}\text { Preoperative hook-wire } \\
\text { marking }\end{array}$} \\
\hline Yes & 13 & 13 & 0 & $<.01$ \\
\hline No & 40 & 20 & 20 & \\
\hline \multicolumn{5}{|l|}{ Palpable GGO } \\
\hline Yes & 19 & 4 & 15 & $<.01$ \\
\hline No & 34 & 29 & 5 & \\
\hline \multicolumn{5}{|l|}{ Operation } \\
\hline Wedge resection & 23 & 18 & 5 & $<.01$ \\
\hline Segmentectomy & 20 & 13 & 7 & \\
\hline Lobectomy & 10 & 2 & 8 & \\
\hline
\end{tabular}

$G G O$, Ground-glass opacity. 
TABLE 2. Internal echo images obtained for different GGO types

\begin{tabular}{lccc}
\hline Internal echo image of GGO & High echo & Low echo & $\boldsymbol{P}$ value \\
\hline Type of GGO in CT & 30 & 3 & .26 \\
$\quad$ Pure GGO & 16 & 4 & \\
$\quad$ Mixed GGO & & & \\
Histologic Noguchi type & 7 & 0 & .39 \\
AAH & 16 & 1 & \\
Type A & 8 & 2 & \\
Type B & 13 & 4 & \\
Type C & & & \\
Deflation of lung & 37 & 0 & $<.01$ \\
$\quad$ Complete & 9 & 7 & \\
$\quad$ Incomplete & $11.5 \pm 4.3$ & $11.9 \pm 4.3$ & .85 \\
CT size of GGO
\end{tabular}

$G G O$, Ground-glass opacity; $C T$, computed tomography; $A A H$, atypical adenomatous hyperplasia.

To achieve a complete collapse of the lung, we devised a method for careful air removal from the airways and applied gentle pressure to the lung surface with an instrumental probe. However, we could not achieve a completely deflated lung in $30 \%$ of the patients examined in the present study, possibly because of trapped sputum in the airway. It is noteworthy in this regard that the cooperation of the presiding anesthesiologist is vital to maintain the lung under entirely deflated conditions.

Although IU proved to be useful for detecting GGO, this procedure has limitations in some patients in terms of its ability to evaluate their tumors. The presence of severe emphysema or asthma has been shown to cause errors in ultrasonographic evaluations. ${ }^{1,12,14}$ It is also often difficult to maintain single-lung ventilation in patients with pulmonary fibrosis or severe heart disease. In the present study, therefore, we did not evaluate pulmonary GGO by IU in patients who had a history of emphysema, asthma, pulmonary fibrosis, or severe heart disease. Such patients will require investigative procedures other than IU to detect and pinpoint their GGO. Hence, we combined a preoperative CT-guided percutaneous hook-wire placement with the IU in 13 patients with pure GGOs of less than 10-mm diameter in size and that were untouched on the visceral pleura. We speculated in these cases also that the target GGO could not be identified by palpation during a VATS operation. We thus contend that for safety purposes, both ultrasonography and other established investigative techniques should be used in patients in whom there is a risk of incomplete lung deflation.

Many GGOs are characterized by a high internal echo and by an acoustic shadow of the posterior echo. This echo pattern might derive from the fact that the tumor is thicker than the normal alveolar wall or may indicate that a delay in air deflation from the GGO has occurred. The presence of a posterior echo might be related to the size of the GGO, but further studies will be necessary to properly assess this. ${ }^{16}$

We propose also from our current findings that ultrasonography is useful for analyzing resected lungs inasmuch
TABLE 3. Characteristics of the posterior echo images obtained for the GGOs analyzed in this study

\begin{tabular}{lcccc}
\hline $\begin{array}{c}\text { Posterior echo } \\
\text { of GGO }\end{array}$ & Attenuation & Accentuating & No change & $\boldsymbol{P}$ value \\
\hline $\begin{array}{l}\text { Deflation of lung } \\
\quad \text { Complete }\end{array}$ & 29 & 0 & 8 & $<.01$ \\
$\quad \begin{array}{l}\text { Incomplete } \\
\text { Internal echo }\end{array}$ & 7 & 7 & 2 & \\
$\quad$ level of GGO & & & & \\
$\quad$ High & 36 & 0 & 10 & $<.01$ \\
$\quad$ Low & 0 & 7 & 0 & \\
Size of GGO & $10.7 \pm 3.3$ & $9.2 \pm 3.5$ & $5.1 \pm 3.0$ & $<.01$ \\
\hline$G G O$, Ground-glass opacity. & & & &
\end{tabular}

as the resection margins determined by IU were comparable with those obtained by histologic examination. GGOs are often difficult to visibly inspect, even in freshly dissected specimens. Hence, ultrasonographic visualization is very helpful in planning the extent of the surgical resection that would be required and also in confirming the surgical margins. In our current study, resection margins could be evaluated successfully by ultrasonography in all 23 patients for whom a wedge partial resection was performed, but could be evaluated in only $50 \%$ of the patients who had undergone a segmentectomy. In our own segmentectomy procedure, we usually reventilate the targeted bronchus before cutting the lung parenchyma so that we can clarify the border of the lung segment. Hence, the distances from the resection margins to the GGO could not be evaluated by IU because of air obstruction around the target GGO in some segmentectomy cases. We contend, therefore, that to enable a proper ultrasonographic evaluation, reventilation into the target lung should be avoided until the lung parenchyma around the target GGO is cut.

In conclusion, we find that IU can safely and effectively localize pulmonary GGO in completely deflated lungs. Moreover, this procedure is useful for the evaluation of surgical margins in a resected lung. This method may thus assist surgeons to perform minimally invasive lung resections, with clear surgical margins, for the treatment of solitary lung GGO.

\section{References}

1. Mattioli S, D’Ovidio F, Daddi N, Ferruzzi L, Pilotti V, Ruffato A, et al. Transthoracic endosonography for the intraoperative localization of lung nodules. Ann Thorac Surg. 2005;79:443-9; discussion 443-9.

2. Goletti O, Buccianti P, Chiarugi M, Pieri L, Sbragia P, Cavina E. Laparoscopic sonography in screening metastases from gastrointestinal cancer: comparative accuracy with traditional procedures. Surg Laparosc Endosc. 1995;5:176-82.

3. Machi J, Sigel B, Zaren HA, Schwartz J, Hosokawa T, Kitamura H, et al. Technique of ultrasound examination during laparoscopic cholecystectomy. Surg Endosc. 1993; 7:544-9.

4. Santambrogio R, Bianchi P, Opocher E, Mantovani A, Schubert L, Ghelma F, et al. Intraoperative ultrasonography (IOUS) during laparoscopic cholecystectomy. Surg Endosc. 1996;10:622-7.

5. Bezzi M, Silecchia G, De Leo A, Carbone I, Pepino D, Rossi P. Laparoscopic and intraoperative ultrasound. Eur J Radiol. 1998;27 Suppl. 2:S207-14.

6. Shennib H, Bret P. Intraoperative transthoracic ultrasonographic localization of occult lung lesions. Ann Thorac Surg. 1993;55:767-9. 
7. Santambrogio R, Montorsi M, Bianchi P, Mantovani A, Ghelma F, Mezzetti M. Intraoperative ultrasound during thoracoscopic procedures for solitary pulmonary nodules. Ann Thorac Surg. 1999;68:218-22.

8. Machi J, Hayashida R, Kurohiji T, Nishimura Y, Edakuni S, Yamashita Y, et al. Operative ultrasonography for lung cancer surgery. J Thorac Cardiovasc Surg. 1989;98:540-5.

9. Tatsumura T. Preoperative and intraoperative ultrasonographic examination as an aid in lung cancer operations. J Thorac Cardiovasc Surg. 1995;110:606-12.

10. Gossot D, Colomer S, Fourquier P, Celerier M, Revillon Y. Thoracoscopic ultrasonic localisation of lung nodules: initial results of an in-vitro and in-vivo study. Endosc Surg Allied Technol. 1994;2:153-5.

11. Mack MJ, Shennib H, Landreneau RJ, Hazelrigg SR. Techniques for localization of pulmonary nodules for thoracoscopic resection. J Thorac Cardiovasc Surg. 1993;106:550-3.
12. Greenfield AL, Steiner RM, Liu JB, Cohn HE, Goldberg BB, Rawool NM, et al. Sonographic guidance for the localization of peripheral pulmonary nodules during thoracoscopy. AJR Am J Roentgenol. 1997;168:1057-60.

13. Yamamoto M, Takeo M, Meguro F, Ishikawa T. Sonographic evaluation for peripheral pulmonary nodules during video-assisted thoracoscopic surgery. Surg Endosc. 2003; 17:825-7.

14. Piolanti M, Coppola F, Papa S, Pilotti V, Mattioli S, Gavelli G. Ultrasonographic localization of occult pulmonary nodules during video-assisted thoracic surgery. Eur Radiol. 2003;13:2358-64.

15. Matsumoto S, Hirata T, Ogawa E, Fukuse T, Ueda H, Koyama T, et al. Ultrasonographic evaluation of small nodules in the peripheral lung during video-assisted thoracic surgery (VATS). Eur J Cardiothorac Surg. 2004;26:469-73.

16. Mikhak Z, Pedersen PC. Acoustic attenuation properties of the lung: an open question. Ultrasound Med Biol. 2002;28:1209-16. 